\title{
Near Complete Laryngopharyngeal Obstruction due to Vegetable Foreign Body in an Infant
}

\author{
Rahul Gupta, ${ }^{1}$ Ashok Gupta ${ }^{2}$
}

\section{Introduction:}

\section{$\underline{\text { ABSTRACT }}$}

Impaction of foreign bodies and obstruction in the upper aero-digestive tract is a medical emergency.

Case Report

A 5-month-old male infant presented with impacted large vegetable foreign body lying in the hypopharynx of an infant causing almost complete laryngeal obstruction. Cardiopulmonary resuscitation was initiated and back blows were given between the blades of the scapula to assist in its removal. Only available curved artery forceps was gently passed beyond the foreign body; blades of the forceps were opened to engage it and foreign body was swept and rolled out. Patient was revived after intensive resuscitation.

\section{Conclusion}

Suspect foreign body in the upper aero-digestive tract in any child presenting with severe respiratory distress or apneic spells or choking. Foreign body may be removed with only available curved artery forceps by gently passing beyond it, followed by opening and engaging blades of the forceps to sweep and roll it out of the upper aero-digestive tract.

Keywords:

Foreign Bodies; Infant; Hypopharynx; Vegetables; Emergencies

$\mathrm{I}$ mpaction of ingested foreign bodies in the upper aero-digestive tract is not a rare paediatric emergency. ${ }^{1}$ Most of the foreign bodies in the paediatric patients present between 1-3 years age group. ${ }^{1}$ Complete or near complete laryngeal obstruction is rarely observed and is associated with high percentage of mortality in children. ${ }^{2,3}$ We report a rare case of an impacted large vegetable foreign body lying in the hypopharynx of an infant causing almost complete laryngeal obstruction, which was managed successfully.

1 - Department of Paediatrics, Kota Medical college, Rajasthan

2 - Department of Paediatrics, SMS Medical College, Jaipur, Rajasthan

\section{Corresponding author:}

Dr Rahul Gupta

email: meetsurgeon007@yahoo.co.in

\section{Case Report}

A 5-month-old male infant presented to the paediatric emergency in an unconscious state with respiratory distress and cyanotic episode. No specific history was available. On examination, patient was unconscious, there was severe respiratory distress, central cyanosis, chest indrawing, pooling of saliva in the mouth, feeble pulse, marked tachycardia and absent air entry on chest auscultation. An urgent direct laryngoscopy was performed in the emergency room to rule out airway obstruction, which revealed a large vegetable foreign body lying in the laryngeal vestibule causing almost complete laryngeal obstruction (Fig.1).

A long curved artery forceps was available in the emergency trolley; it was negotiated beyond the foreign body and an attempt was made to remove it. As the foreign body looked spherical and was impacted in the hypopharynx, attempt failed. Emergency OT was informed for emergency tracheotomy, but, within few seconds patient became apneic with bradycardia. 


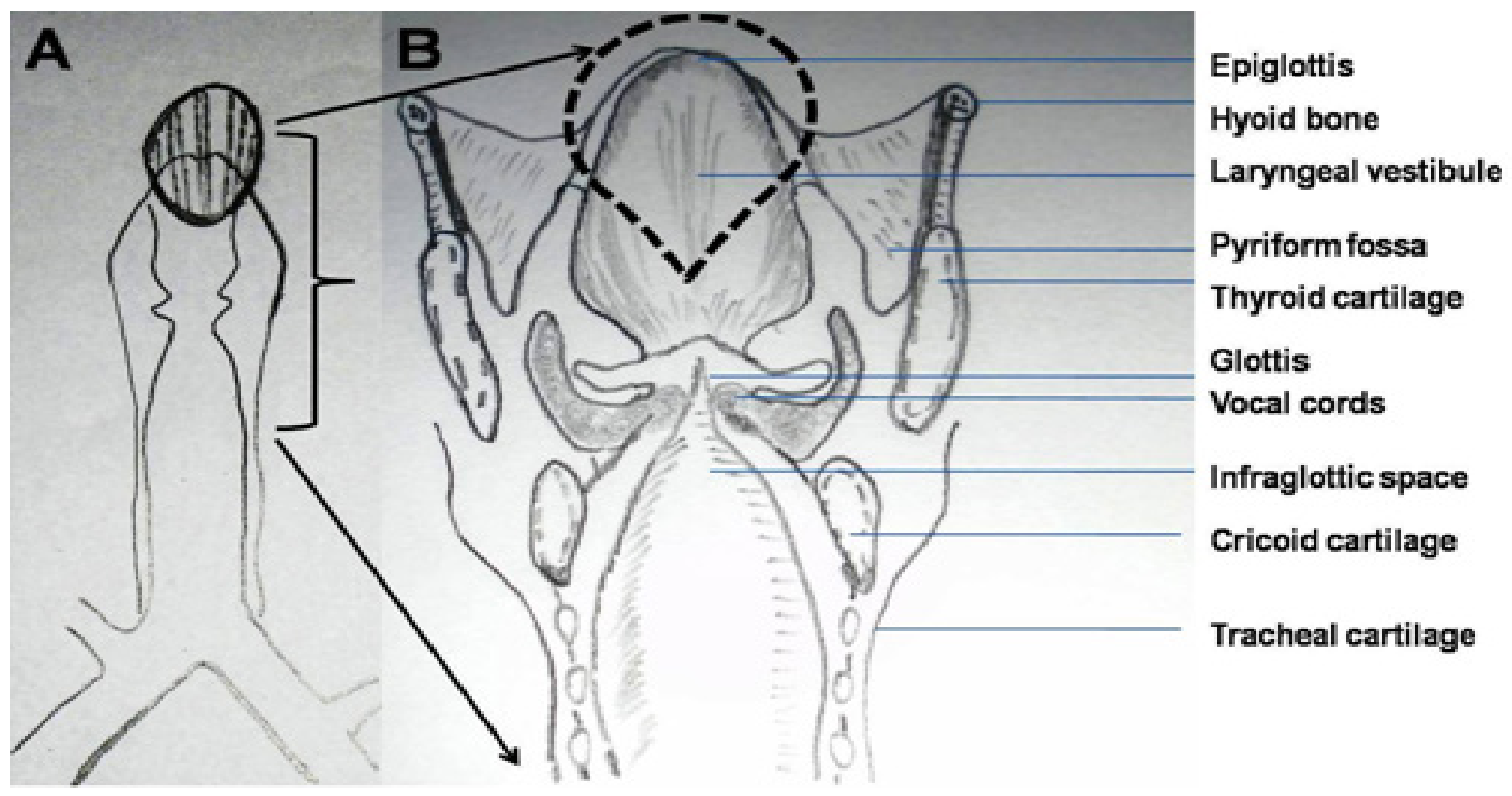

Fig.1: Diagrammatic representation showing oval vegetable foreign body (Kachri) impacted in the laryngeal vestibule causing near complete hypopharyngeal obstruction.

Cardiopulmonary resuscitation (CPR) was initiated; back blows were given between the blades of the scapula, both in prone (Fig.2) and upside down position to assist in its removal.

A final attempt was made to remove it with same artery forceps, before performing an emergency tracheotomy. The artery forceps was again gently passed beyond the foreign body; blades of the forceps were opened to engage it and foreign body was swept and rolled out of the laryngeal vestibule and finally removed. Inspection revealed average sized vegetable (Kachri) foreign body (Fig.3). Patient was intubated with endotracheal tube no.3.5, CPR was reinitiated and patient was revived with advanced cardiac life support system. Ventilatory support was initiated and patient was shifted to ICU for critical care. The patient was gradually weaned from the ventilatory support. Radiographs were performed followed by Computed Tomography (CT) of the chest to rule out any pneumothorax, aspiration pneumonitis and to detect any residual foreign body. Bronchoscopy was done to recheck presence of any residual foreign body. The patient was started on breast feeds, observed for 2 days and was discharged. He is doing well on followup. On further enquiring, mother revealed that patients elder sister was playing with the vegetable and during fun activity, unknowingly she put it in patient's mouth.

\section{Discussion}

Impaction of foreign bodies in the upper aero-digestive tract and airway obstruction is a medical emergency requiring urgent intervention to save the patient's life..$^{1-3}$ Children between the age groups of 1-3 years are most susceptible, ${ }^{1}$ while it is rare in infants below 6 months of age group. ${ }^{1-4}$ Choking is commonly seen in young children below 5 years of age because their swallow functions are inadequate, usually putting things in their mouth to recognize them, inability to appreciate the size of a piece of food, small airways, inadequate dentition for chewing and weaker cough reflex. ${ }^{1-4}$

Foreign bodies of vegetable origin in the upper aero-digestive tract and laryngo-tracheobronchial tree are common in children less than 4 years of age. The most common vegetable foreign bodies among the paediatric patients being peanut, and its shell, or bits of peanut), seeds and fruits. ${ }^{1}$ Other commonly seen are coins, toy parts, stones, bones, screws, button batteries and nails. ${ }^{1-4}$ In the present case the foreign body was 


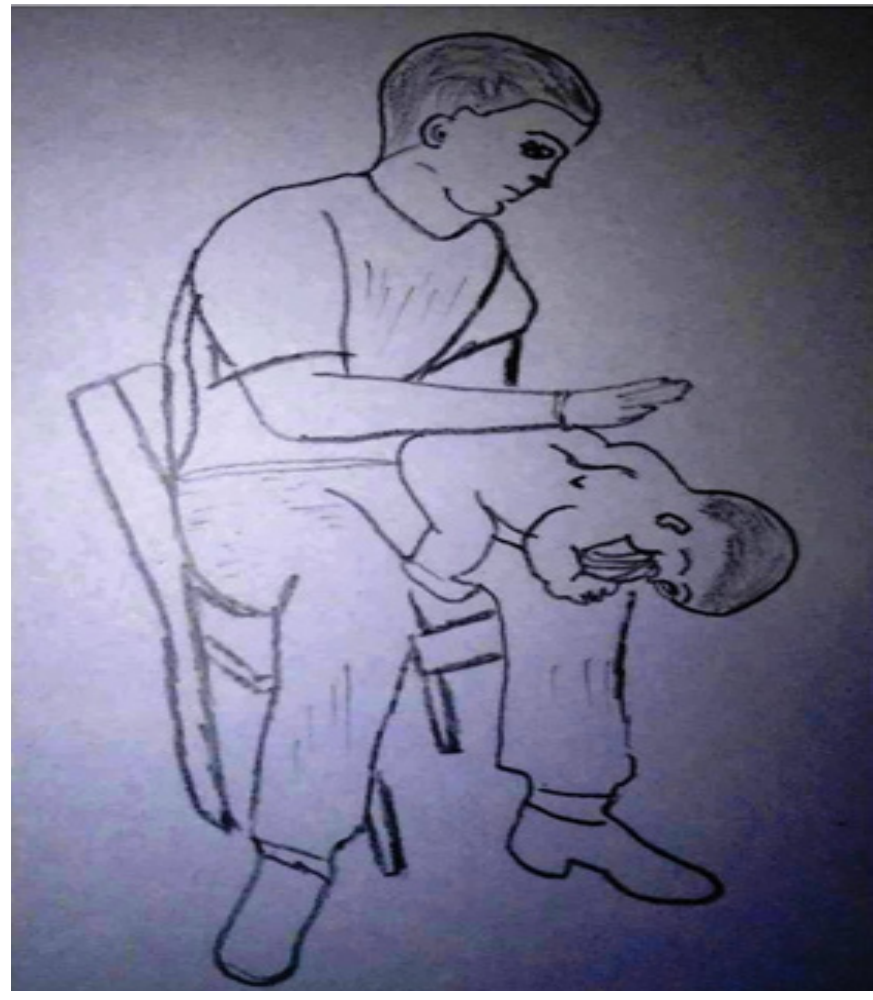

Fig.2. Diagrammatic representation showing right method of performing back blow maneuvers with heel of the hands between the shoulder blades in a conscious infant, who is being held prone on rescuer's forearm with head down and jaw tightly supported by hand.

Cucumis Callosus or Cucumis melo sp agrestis. It is a fruit, ovoid in shape, found in Rajasthan and western India and commonly known as Kachri or Kaachri. It is a wild variety of cucumbers and looks like miniature watermelon. It has numerous seeds and little flesh inside. Other names are wild melon, wild musk melon, and small gourd. It is rarely seen in other parts of India. It was large $(4 \mathrm{cms})$ for the size of the infant (5months) and is rarely reported in the literature.

Presentation and damage incurred by the impacted foreign body varies according to its site, size, shape and consistency. ${ }^{5}$ Prior surgery for esophageal atresia, anastomotic stricture, esophageal stenosis are important considerations. Preceding history of playing with small items just before the onset of symptoms may or may not be available. ${ }^{3-4}$ In our case, the baby was brought by a passerby who saw the mother of the child weeping for
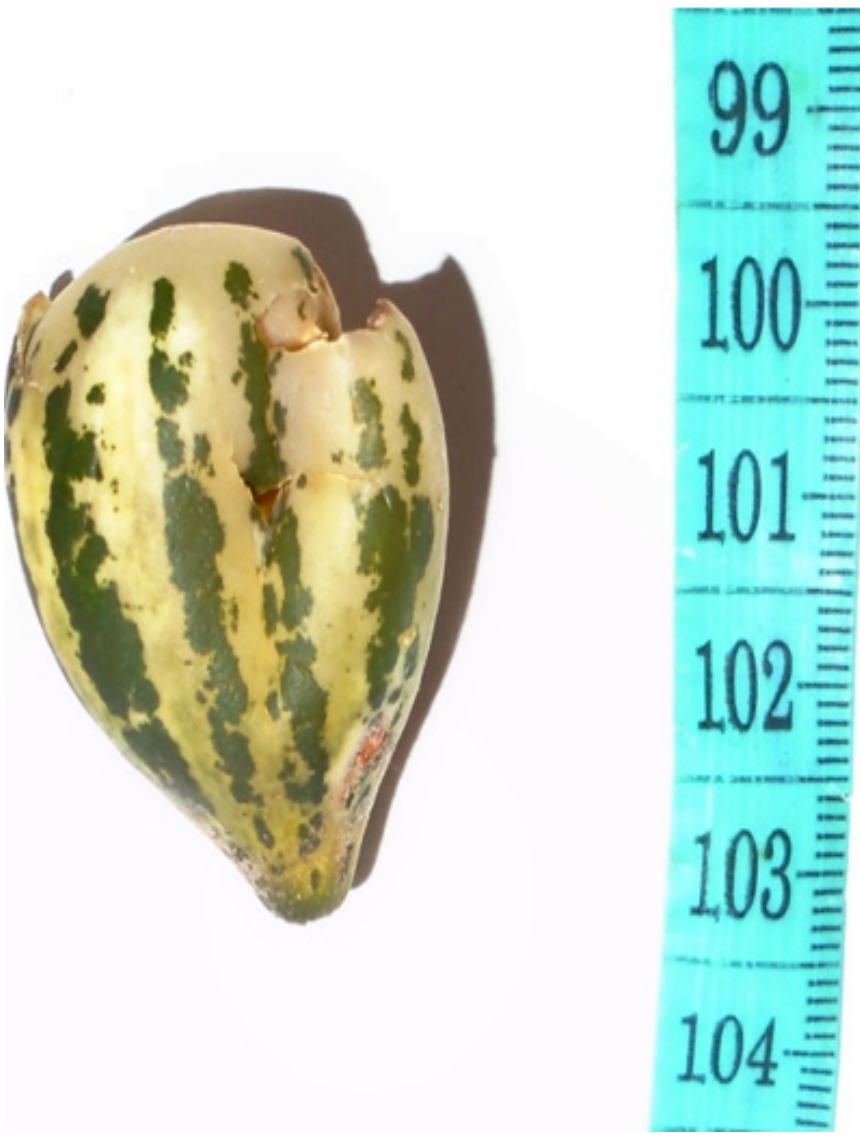

Fig.3. Kachri (vegetable foreign body) which was removed successfully from the hypopharynx.

his baby, as he was having severe breathing difficulty.

Paediatric patients especially infants may present with inability to breathe, respiratory distress, choking, cyanosis and stridor. Older children may complain of coughing, hemoptysis, intractable asthma, change in voice quality, inability to speak, neck pain and swallowing problems. ${ }^{5-6}$ In our case the patient was unconscious with severe respiratory distress, cyanosis and apneic spells. A high degree of suspicion and evaluation is required even if there is a single clue pointing towards foreign body aspiration. Usually, history of aspiration, clinical examination and plain radiogram (lateral and antero-posterior) of neck and chest is diagnostic.

Complete blockage of the airway is not required as sudden death due to laryngeal spasm can occur by small object with partial obstruction. ${ }^{7}$ Urgent respiratory resuscitation and treatment is required in case of 
complete airway obstruction to prevent anoxic cerebral injury and unfavourable outcome..$^{1-7}$ Initial management in a conscious infant with choking consists of back blows and chest thrusts. ${ }^{8}$ The infant is held prone on rescuer's forearm with head down and jaw, head tightly supported by hand. Five back blows with heel of the hands between the shoulder blades are given (Fig.2), followed by five chest thrusts in supine position. ${ }^{8}$ While in older children five back blows with five abdominal thrusts are given, but, abdominal thrusts are contraindicated in infants. The sequence is repeated until obstruction is relieved or patient becomes unconscious. ${ }^{8}$ This maneuver alone may lead to expulsion of the foreign body. ${ }^{2}$ We believe that in our case, back blows maneuver partly dislodged the impacted foreign body in the hypopharynx, which led to its removal using artery forceps.

In an unconscious child with choking, mouth is opened and attempt should be made to remove it. Removal with the help of curved laryngeal forceps/Magill forceps is recommended. This is known as Magill forceps technique. Its use has been associated with the improved outcome in emergency management of life threatening hypopharyngeal obstruction. ${ }^{9}$ Blind finger sweep should not be performed in a conscious patient; finger sweep should only be performed for foreign bodies that can be seen and in an unconscious patient. ${ }^{3}$ CPR should start if unsuccessful. ${ }^{8}$ In our case, direct laryngoscopy was performed and removal was performed with help of available curved artery forceps. Emergency tracheotomy is required when there is failure of successful removal in case of large foreign bodies with completely blocked airway. ${ }^{1}$ For the foreign bodies below the level of vocal cord, pushing the foreign body into the right main bronchus and restoring the ventilation by the left lung has been mentioned. ${ }^{10}$

Most of time, impacted foreign bodies in the upper airway are relatively small and patient is hemodynamically stable. These are routinely removed by direct endoscopy (Macintosh laryngoscope) using Magill forceps technique or rigid bronchoscope (instrument of choice) under general anesthesia. ${ }^{1,4,5,10}$ Some centers perform indirect fiber-optic bronchoscopy with adequate local anesthesia. ${ }^{4}$

Chest radiographs and computed tomography should be done to detect foreign body and rule out presence of secondary complications like pneumonia, mediastinitis, empyema, obstructive emphysema, obstructive atelectasis, pneumothorax and mediastinal emphysema. ${ }^{1}$ These complications are seen with incomplete obstruction. Missed pneumothorax has been reported to cause mortality in one study. ${ }^{1}$ Airway obstruction results in high alveolar pressure and rupture followed by interstitial, mediastinal and subcutaneous emphysema. ${ }^{1}$

ICU care is mandatory to treat chemical pneumonia especially in vegetable foreign bodies due to presence of its fat content. ${ }^{1}$ Antibiotics and chest physiotherapy are given to prevent infection and removal of secretions respectively. In our case, after successful removal proper evaluation was done by performing check bronchoscopy and $\mathrm{CT}$ to rule out residual foreign (may be multiple) body. Educating parents about the possible objects which lead to such catastrophic event and minimizing the complications by mass education for management of choking due to foreign body in the upper aero-digestive tract is very important.

\section{Conclusion}

Clinicians should suspect foreign body in the upper aero-digestive tract in any child presenting with severe respiratory distress or apneic spells or choking and carrying urgent intervention for favorable outcome. Initial management in a conscious infant with choking consists of back blows and chest thrusts. Abdominal thrusts are contraindicated in infants. In case of extreme emergency, foreign body may be removed with only available curved artery forceps by gently passing beyond it, followed by opening and engaging blades of the forceps to sweep and roll it out of the upper aerodigestive tract.

\section{References}

1. Narasimhan KL, Chowdhary SK, Suri S, Mahajan JK, Samujh R, Rao KLN. Foreign body airway obstructions in childrenlessons learnt from a prospective audit. J Indian Assoc Pediatr Surg. 2002; 7:184-9

2. Gencpinar P, Duman M. Importance of back blow maneuvers in a 6 month old patient with sudden upper airway obstruction. Turk J Emerg Med. 2015; 15(4):177-8 
3. Abder-Rahman HA. Infants choking following blind finger sweep. J Pediatr. (Rio J) 2009; 85(3):273-5

4. Kuo CL. Management Strategies for Ingestion of Foreign Objects in the Laryngopharynx. J Oto Rec Surg. 2015; 1(2):110

5. Wiseman NE. The diagnosis of foreign body aspiration in childhood. J Pediatr Surg. 1984; 19(5):531-5

6. Heimlich HJ. A life-saving maneuver to prevent food choking. J Am Med Assoc. 1975; 234(4):398-401

7. Nayak SR, Jena MK, Panda BB, Singh P. Sudden Deaths Due to Choking. J Indian Acad Forensic Med. 2015; 37(1):106-8

8. Berg MD, Schexnayder SM, Chameides L, Terry M, Donoghue
A, Hickey RW, et al. Pediatric basic life support: 2010 American Heart Association Guidelines for Cardiopulmonary Resuscitation and Emergency Cardiovascular Care. Pediatr. 2010; 126:1345-60

9. Sakai T, Kitamura T, Iwami T, Nishiyama C, TanigawaSugihara K, Hayashida S, et al. Effectiveness of prehospital Magill forceps use for out-of-hospital cardiac arrest due to foreign body airway obstruction in Osaka City. Scand J Trauma Resusc Emerg Med. 2014; 22:53

10. Pfleger A, Eber E. Management of acute severe upper airway obstruction in children. Paediatr Respir Rev. 2013;14:70-7. 